\title{
A fixed point theorem for Boolean networks expressed in terms of forbidden subnetworks
}

\author{
Adrien Richard $\|^{\dagger}$ \\ Laboratoire I3S, CNRS \& Université de Nice-Sophia Antipolis, France
}

\begin{abstract}
We are interested in fixed points in Boolean networks, i.e. functions $f$ from $\{0,1\}^{n}$ to itself. We define the subnetworks of $f$ as the restrictions of $f$ to the hypercubes contained in $\{0,1\}^{n}$, and we exhibit a class $\mathcal{F}$ of Boolean networks, called even or odd self-dual networks, satisfying the following property: if a network $f$ has no subnetwork in $\mathcal{F}$, then it has a unique fixed point. We then discuss this "forbidden subnetworks theorem". We show that it generalizes the following fixed point theorem of Shih and Dong: if, for every $x$ in $\{0,1\}^{n}$, there is no directed cycle in the directed graph whose the adjacency matrix is the discrete Jacobian matrix of $f$ evaluated at point $x$, then $f$ has a unique fixed point. We also show that $\mathcal{F}$ contains the class $\mathcal{F}^{\prime}$ of networks whose the interaction graph is a directed cycle, but that the absence of subnetwork in $\mathcal{F}^{\prime}$ does not imply the existence and the uniqueness of a fixed point.
\end{abstract}

Keywords: Boolean network, fixed point, self-dual Boolean function, discrete Jacobian matrix, feedback circuit.

\section{Introduction}

A function $f$ from $\{0,1\}^{n}$ to itself is often seen as a Boolean network with $n$ components. On on hand, the dynamics of the network is described by the iterations of $f$; for instance, with the synchronous iteration scheme, the dynamics is described by the recurrence $x^{t+1}=f\left(x^{t}\right)$. On the other hand, the "structure" of the network is described by a directed graph $G(f)$ : the vertices are the $n$ components, and there exists an arc from $j$ to $i$ when the evolution of the $i$ th component depends on the evolution of the $j$ th one.

Boolean networks have many applications. In particular, from the seminal works of Kauffman (1969) and Thomas (1973), they are extensively used to model gene networks. In most cases, fixed points are of special interest. For instance, in the context of gene networks, they are often seen as stable patterns of gene expression at the basis of particular biological processes.

In this paper, we are interested in sufficient conditions for the existence and the uniqueness of a fixed point for $f$. Such a condition was first obtained by Robert (1980), who proved that if $G(f)$ has no directed cycle, then $f$ has a unique fixed point. This result was then generalized by Shih and Dong (2005). They associated to each point $x$ in $\{0,1\}^{n}$ a local interaction graph $G f(x)$, which is a subgraph of $G(f)$ defined as the directed graph whose the adjacency matrix is the discrete Jacobian matrix of $f$ evaluated at point $x$, and they proved that if $G f(x)$ has no directed cycle for all $x$ in $\{0,1\}^{n}$, then $f$ has a unique fixed point. Up

\footnotetext{
${ }^{\dagger}$ Email: richardeunice.fr. 
to our knowledge, this is the weakest condition known to be sufficient for the presence and the uniqueness of a fixed point.

In this paper, we establish a sufficient condition for the existence and the uniqueness of a fixed point that is not expressed in terms of directed cycles. In Section 2, we defined, in a natural way, the subnetworks of $f$ as the restrictions of $f$ to the hypercubes contained in $\{0,1\}^{n}$, and we introduce the class $\mathcal{F}$ of even and odd self-dual networks. In Section 3 , we prove the main result: if $f$ has no subnetworks in $\mathcal{F}$, then it has a unique fixed point. The rest of the paper discusses this "forbidden subnetworks theorem". In section 4, we show that it generalizes the fixed point theorem of Shih and Dong mentioned above. In section 5 , we study the effect of the absence of subnetwork in $\mathcal{F}$ on the asynchronous state graph of $f$, which is a directed graph on $\{0,1\}^{n}$ constructed from the asynchronous iterations of $f$ and proposed by Thomas (1973) as a model for the dynamics of gene networks. Finally, in Section 6 , we compare $\mathcal{F}$ with the well-known class $\mathcal{F}^{\prime}$ of networks $f$ whose the interaction graph $G(f)$ is a directed cycle. Mainly, we show that $\mathcal{F}^{\prime} \subseteq \mathcal{F}$ and that the absence of subnetwork in $\mathcal{F}^{\prime}$ is not sufficient for the existence and the uniqueness of a fixed point.

\section{Definitions and notations}

In this section, we introduce the definitions needed to state and prove the main result. Let $\mathbb{B}=\{0,1\}$, let $n$ be a positive integer, let $[n]=\{1, \ldots, n\}$, and let $i \in[n]$. The $i$ th unit vector of $\mathbb{B}^{n}$ is denoted $e_{i}$ (all the components are 0 , excepted the $i$ th one which is 1 ). The sum modulo two is denoted $\oplus$. It is applied componentwise on elements of $\mathbb{B}^{n}$ : for all $x, y \in \mathbb{B}^{n}$,

$$
x \oplus y=\left(x_{1} \oplus y_{1}, \ldots, x_{n} \oplus y_{n}\right) \quad \text { and } \quad x \oplus 1=\left(x_{1} \oplus 1, \ldots, x_{n} \oplus 1\right) .
$$

Hence, $x \oplus 1$ may be seen as the negation of $x$. The number of ones that $x$ contains is denoted $\|x\|$, i.e. $\|x\|=\sum_{i=1}^{n} x_{i}$. Thus $\|x \oplus y\|$ gives the Hamming distance between two points $x$ and $y$ of $\mathbb{B}^{n}$. We say that $x$ is even (odd) if $\|x\|$ is even (odd) (there exists $2^{n-1}$ even (odd) points in $\mathbb{B}^{n}$ ). The point of $\mathbb{B}^{n}$ obtained from $x$ by assigning the $i$ th component to $\alpha \in \mathbb{B}$ is denoted $x^{i \alpha}$, i.e.

$$
x^{i \alpha}=\left(x_{1}, \ldots, x_{i-1}, \alpha, x_{i+1}, \ldots, x_{n}\right) .
$$

If $n>1$, the point of $\mathbb{B}^{n-1}$ obtained from $x$ be removing the $i$ th component is denoted $x_{-i}$, i.e.

$$
x_{-i}=\left(x_{1}, \ldots, x_{i-1}, x_{i+1}, \ldots, x_{n}\right) .
$$

We call ( $n$-dimensional Boolean) networks any function $f$ from $\mathbb{B}^{n}$ to itself.

Definition 1 (Conjugate) The conjugate of $f: \mathbb{B}^{n} \rightarrow \mathbb{B}^{n}$ is the following n-dimensional network:

$$
\tilde{f}: \mathbb{B}^{n} \rightarrow \mathbb{B}^{n}, \quad \tilde{f}(x)=x \oplus f(x) \quad \forall x \in \mathbb{B}^{n} .
$$

Remark that $\tilde{f}(x)=0$ if and only if $x$ is a fixed point of $f$, i.e. $f(x)=x$.

Definition 2 (Self-dual networks and even/odd networks) $f$ is self-dual if

$$
f(x)=f(x \oplus 1) \oplus 1 \quad \forall x \in \mathbb{B}^{n} .
$$

$f$ is even (odd) if the image of $\tilde{f}$ is the set of even points of $\mathbb{B}^{n}$, i.e.

$$
\left\{\tilde{f}(x) \mid x \in \mathbb{B}^{n}\right\}=\left\{x \mid x \in \mathbb{B}^{n} \text { and }\|x\| \text { is even (odd) }\right\} .
$$


We say that $f$ is even (odd) self-dual if it is both even (odd) and self-dual. Note that $f(x)=f(x \oplus 1) \oplus 1$ if and only if $\tilde{f}(x \oplus 1)=\tilde{f}(x)$. Note also that if $f$ is even (odd) self-dual, then for each even (odd) point $x \in \mathbb{B}^{n}$, the preimage of $x$ by $\tilde{f}$ is of cardinality two, i.e. there exists exactly two distinct points $y, z \in \mathbb{B}^{n}$ such that $\tilde{f}(y)=\tilde{f}(z)=x$. Since $\tilde{f}(x)=0$ if and only if $f(x)=x$, we deduce that if $f$ is even self-dual, then it has exactly two fixed points (obviously, if $f$ is odd self-dual, then it has no fixed point).

Definition 3 (Immediate subnetworks) If $n>1, \alpha \in \mathbb{B}$ and $i \in[n]$, we call immediate subnetwork of $f$ (obtained by fixing the $i$ th component to $\alpha$ ) the following $(n-1)$-dimensional network:

$$
f^{i \alpha}: \mathbb{B}^{n-1} \rightarrow \mathbb{B}^{n-1}, \quad f^{i \alpha}\left(x_{-i}\right)=f\left(x^{i \alpha}\right)_{-i} \quad \forall x \in \mathbb{B}^{n} .
$$

Remark that conjugate of $f^{i \alpha}$ is equal to the immediate subnetwork $\tilde{f}^{i \alpha}$ of the conjugate $\tilde{f}$ of $f$ :

$$
\widetilde{f^{i \alpha}}\left(x_{-i}\right)=x_{-i} \oplus f^{i \alpha}\left(x_{-i}\right)=x_{-i} \oplus f\left(x^{i \alpha}\right)_{-i}=\left(x \oplus f\left(x^{i \alpha}\right)\right)_{-i}=\tilde{f}\left(x^{i \alpha}\right)_{-i}=\tilde{f}^{i \alpha}\left(x_{-i}\right) .
$$

Definition 4 (Subnetworks) The subnetworks of $f$ are inductively defined by: (1) if $n=1$, then $f$ has a unique subnetwork, which is $f$ itself; and (2) if $n>1$, the subnetworks of $f$ are $f$ and the subnetworks of the immediate subnetworks of $f$. A strict subnetwork of $f$ is a subnetwork of $f$ different than $f$.

\section{Main result}

Theorem 1 (Forbidden subnetworks theorem) If a network $f: \mathbb{B}^{n} \rightarrow \mathbb{B}^{n}$ has no even or odd self-dual subnetwork, then the conjugate of $f$ is a bijection, and in particular, $f$ has a unique fixed point.

The proof of Theorem 1 needs the following two lemmas.

Lemma 1 Let $X$ be a non-empty subset of $\mathbb{B}^{n}$ and $V(X)=\left\{x \oplus e_{i} \mid x \in X, i \in[n]\right\}$. If $X$ and $V(X)$ are disjoint and $|X| \geq|V(X)|$, then $X$ is either the set of even points of $\mathbb{B}^{n}$ or the set of odd points of $\mathbb{B}^{n}$.

Proof: by induction on $n$. The case $n=1$ is obvious. So suppose that $n>1$ and that the lemma holds for the dimensions less than $n$. Let $X$ be a non-empty subset of $\mathbb{B}^{n}$ satisfying the conditions of the statement. Let $\alpha \in \mathbb{B}$, and consider the following subsets of $\mathbb{B}^{n-1}$ :

$$
X^{\alpha}=\left\{x_{-n} \mid x \in X, x_{n}=\alpha\right\}, \quad V(X)^{\alpha}=\left\{x_{-n} \mid x \in V(X), x_{n}=\alpha\right\} .
$$

We first prove that $V\left(X^{\alpha}\right) \subseteq V(X)^{\alpha}$ and $X^{\alpha} \cap V\left(X^{\alpha}\right)=\emptyset$. Let $x \in \mathbb{B}^{n}$ with $x_{n}=\alpha$ be such that $x_{-n} \in V\left(X^{\alpha}\right)$. To prove that $V\left(X^{\alpha}\right) \subseteq V(X)^{\alpha}$, it is sufficient to prove that $x_{-n} \in V(X)^{\alpha}$. Since $x_{-n} \in V\left(X^{\alpha}\right)$, there exists $y \in \mathbb{B}^{n}$ with $y_{n}=\alpha$ and $i \in[n-1]$ such that $y_{-n} \in X^{\alpha}$ and $x_{-n}=y_{-n} \oplus e_{i}$. So $x=y \oplus e_{i}$, and since $y_{n}=\alpha$, we have $y \in X$. Hence $x \in V(X)$ and since $x_{n}=\alpha$, we have $x_{-n} \in V(X)^{\alpha}$. We now prove that $X^{\alpha} \cap V\left(X^{\alpha}\right)=\emptyset$. Indeed, otherwise, there exists $x \in \mathbb{B}^{n}$ with $x_{n}=\alpha$ such that $x_{-n} \in X^{\alpha} \cap V\left(X^{\alpha}\right)$. Since $V\left(X^{\alpha}\right) \subseteq V(X)^{\alpha}$, we have $x_{-n} \in X^{\alpha} \cap V(X)^{\alpha}$, and since $x_{n}=\alpha$, we deduce that $x \in X \cap V(X)$, a contradiction.

Now, since $V\left(X^{\alpha}\right) \subseteq V(X)^{\alpha}$, we have

$$
|X|=\left|X^{0}\right|+\left|X^{1}\right| \geq|V(X)|=\left|V(X)^{0}\right|+\left|V(X)^{1}\right| \geq\left|V\left(X^{0}\right)\right|+\left|V\left(X^{1}\right)\right| .
$$

So $\left|X^{0}\right| \geq\left|V\left(X^{0}\right)\right|$ or $\left|X^{1}\right| \geq\left|V\left(X^{1}\right)\right|$. Suppose that $\left|X^{0}\right| \geq\left|V\left(X^{0}\right)\right|$, the other case being similar. Since $X^{0} \cap V\left(X^{0}\right)=\emptyset$, by induction hypothesis $X^{0}$ is either the set of even points of $\mathbb{B}^{n-1}$ or the 
set of odd points of $\mathbb{B}^{n-1}$. So in both cases, we have $\left|X^{0}\right|=\left|V\left(X^{0}\right)\right|=2^{n-1}$. We deduce that $\left|X^{1}\right| \geq\left|V\left(X^{1}\right)\right|$, and so, by induction hypothesis, $X^{1}$ is either the set of even points of $\mathbb{B}^{n-1}$ or the set of odd points of $\mathbb{B}^{n-1}$. But $X^{0}$ and $X^{1}$ are disjointed: for all $x \in \mathbb{B}^{n}$, if $x_{-n} \in X^{0} \cap X^{1}$, then $x^{n 0}$ and $x^{n 1}$ are two points of $X$, and $x^{n 1}=x^{n 0} \oplus e_{n} \in V(X)$, a contradiction. So if $X^{0}$ is the set of even (odd) points of $\mathbb{B}^{n-1}$, then $X^{1}$ is the set of odd (even) points of $\mathbb{B}^{n-1}$, and we deduce that $X$ is the set of even (odd) points of $\mathbb{B}^{n}$.

Lemma 2 Let $f: \mathbb{B}^{n} \rightarrow \mathbb{B}^{n}$. Suppose that the conjugate of every immediate subnetwork of $f$ is a bijection. If the conjugate of $f$ is not a bijection, then $f$ is even or odd self-dual.

Proof: Suppose that $f$ satisfies the conditions of the statement, and that the conjugate $\tilde{f}$ of $f$ is not a bijection. Let $\tilde{X} \subseteq \mathbb{B}^{n}$ be the image of $\tilde{f}$, and let $X=\mathbb{B}^{n} \backslash \tilde{X}$. Since $\tilde{f}$ is not a bijection, $X$ is not empty. We first prove the following property:

(*) For every $x \in X$ and $i \in[n]$, the preimage of $x \oplus e_{i}$ by $\tilde{f}$ is of cardinality two.

Let $x \in X$ and $i \in[n]$. By hypothesis, the conjugate of $f^{i 0}$ is a bijection, so there exists a unique point in $\mathbb{B}^{n-1}$ whose the image by $\tilde{f}^{i 0}$ is $x_{-i}$. We deduce that there exists a unique point $y \in \mathbb{B}^{n}$ such that $y_{i}=0$ and $\tilde{f}^{i 0}\left(y_{-i}\right)=x_{-i}$. Then, $\tilde{f}(y)_{-i}=\tilde{f}\left(y^{i 0}\right)_{-i}=\tilde{f}^{i 0}\left(y_{-i}\right)=x_{-i}$. We deduce that either $\tilde{f}(y)=x$ or $\tilde{f}(y)=x \oplus e_{i}$. Since $x \in X$ we have $\tilde{f}(y) \neq x$ so $\tilde{f}(y)=x \oplus e_{i}$. Hence, we have proved that there exists a unique point $y \in \mathbb{B}^{n}$ such that $y_{i}=0$ and $\tilde{f}(y)=x \oplus e_{i}$, and we prove with similar arguments that there exists a unique point $z \in \mathbb{B}^{n}$ such that $z_{i}=1$ and $\tilde{f}(z)=x \oplus e_{i}$. This proves $(*)$.

We are now in position to prove that $f$ is even or odd. Let $V(X)=\left\{x \oplus e_{i} \mid x \in X, i \in[n]\right\}$. We have

$$
|X|+|\tilde{X}|=2^{n}=\left|\tilde{f}^{-1}(\tilde{X})\right|=\left|\tilde{f}^{-1}(V(X))\right|+\left|\tilde{f}^{-1}(\tilde{X} \backslash V(X))\right| \geq\left|\tilde{f}^{-1}(V(X))\right|+|\tilde{X} \backslash V(X)| .
$$

Following (*), we have $\left|\tilde{f}^{-1}(V(X))\right|=2|V(X)|$ and $V(X) \subseteq \tilde{X}$, so

$$
|X|+|\tilde{X}| \geq 2|V(X)|+|\tilde{X} \backslash V(X)|=2|V(X)|+|\tilde{X}|-|V(X)|=|V(X)|+|\tilde{X}| .
$$

Therefore, $|X| \geq|V(X)|$, and since $V(X) \subseteq \tilde{X}=\mathbb{B}^{n} \backslash X$, we have $X \cap V(X)=\emptyset$. So according to Lemma $1, X$ is either the set of even points of $\mathbb{B}^{n}$ or the set of odd points of $\mathbb{B}^{n}$. We deduce that in the first (second) case, $\tilde{X}$ is the set of odd (even) points of $\mathbb{B}^{n}$. Thus, $f$ is even or odd.

It remains to prove that $f$ is self-dual. Let $x \in \mathbb{B}^{n}$. For all $i \in[n]$, since $\|\tilde{f}(x)\|$ and $\left\|\tilde{f}(x) \oplus e_{i}\right\|$ have not the same parity, and since $f$ is even or odd, we have $\tilde{f}(x) \oplus e_{i} \in X$. Thus, according to (*), the preimage of $\left(\tilde{f}(x) \oplus e_{i}\right) \oplus e_{i}=\tilde{f}(x)$ by $\tilde{f}$ is of cardinality two. Consequently, there exists a point $y \in \mathbb{B}^{n}$, distinct from $x$, such that $\tilde{f}(y)=\tilde{f}(x)$. Let us proved that $x=y \oplus 1$. Indeed, if $x_{i}=y_{i}=0$ for some $i \in[n]$, then $\tilde{f}^{i 0}\left(x_{-i}\right)=\tilde{f}(x)_{-i}=\tilde{f}(y)_{-i}=\tilde{f}^{i 0}\left(y_{-i}\right)$. Since $x \neq y$, we deduce that $\tilde{f}^{i 0}$ is not a bijection, a contradiction. We show similarly that if $x_{i}=y_{i}=1$, then $\tilde{f}^{i 1}$ is not a bijection. So $x=y \oplus 1$. Consequently, $\tilde{f}(x \oplus 1)=\tilde{f}(x)$, and we deduce that $f$ is self-dual.

Proof of Theorem 1; by induction on $n$. The case $n=1$ is obvious. So suppose that $n>1$ and that the theorem holds for the dimensions less than $n$. Suppose that $f$ has no even or odd self-dual subnetwork. Under this condition, $f$ is neither even self-dual nor odd self-dual (since $f$ is a subnetwork of $f$ ), and every immediate subnetwork of $f$ has no even or odd self-dual subnetwork. So, by induction hypothesis, the dual of every strict subnetwork of $f$ is a bijection, and we deduce from Lemma 2 that the dual of $f$ is a 
bijection. Thus, in particular, there exists a unique point $x \in \mathbb{B}^{n}$ such that $\tilde{f}(x)=0$, and since $\tilde{f}(x)=0$ if and only if $f(x)=x$, this point $x$ is the unique fixed point of $f$.

Clearly, if $f$ has no even or odd self-dual subnetwork, then every subnetwork of $f$ has no even or odd self-dual subnetwork, and according to Theorem 1 , the conjugate of every subnetwork of $f$ is a bijection. Conversely, if the conjugate of every subnetwork of $f$ is a bijection, then $f$ has no even or odd self-dual subnetwork, since the conjugate of an even or odd self-dual network is not a bijection. Consequently, we have the following characterization:

Corollary 1 The conjugate of each subnetwork of $f$ is a bijection if and only if $f$ has no even or odd self-dual network.

Example $1 f: \mathbb{B}^{3} \rightarrow \mathbb{B}^{3}$ is defined by:

$$
f\left(x_{1}, x_{2}, x_{3}\right)=\left(\overline{x_{2}} \wedge x_{3}, \overline{x_{3}} \wedge x_{1}, \overline{x_{1}} \wedge x_{2}\right) .
$$

Remark that $f$ is not self-dual, since $f(000)=f(111)=000$. The immediate subnetworks of $f$ are:

$$
\begin{aligned}
& f^{10}\left(x_{2}, x_{3}\right)=\left(0, x_{2}\right) \\
& f^{11}\left(x_{2}, x_{3}\right)=\left(\overline{x_{3}}, 0\right) \\
& f^{20}\left(x_{1}, x_{3}\right)=\left(x_{3}, 0\right) \\
& f^{21}\left(x_{1}, x_{3}\right)=\left(0, \overline{x_{1}}\right) \\
& f^{30}\left(x_{1}, x_{2}\right)=\left(0, x_{1}\right) \\
& f^{31}\left(x_{1}, x_{2}\right)=\left(\overline{x_{2}}, 0\right)
\end{aligned}
$$

So each immediate subnetwork $f^{i \alpha}$ of $f$ has one component fixed to zero, and so is not self-dual. Futhermore, each immediate subnetwork of $f^{i \alpha}$ is the one dimensional network $h$ defined by $h(0)=h(1)=0$, which is not self-dual. So $f$ has no self-dual subnetwork, and we deduce from Theorem 1 that the conjugate of $\tilde{f}$ of $f$ is a bijection, and that $f$ has a unique fixed point. Indeed:

\begin{tabular}{ccc}
$x$ & $f(x)$ & $\tilde{f}(x)$ \\
\hline 000 & 000 & 000 \\
001 & 100 & 101 \\
010 & 001 & 011 \\
011 & 001 & 010 \\
100 & 010 & 110 \\
101 & 100 & 001 \\
110 & 010 & 100 \\
111 & 000 & 111
\end{tabular}

\section{Remarks on the theorem of Shih and Dong}

In this section, we show that Theorem 1 implies a fixed point theorem due to Shih and Dong (2005). In order to state this theorem, we need additional definitions. Let

$$
f: \mathbb{B}^{n} \rightarrow \mathbb{B}^{n}, \quad f(x)=\left(f_{1}(x), \ldots, f_{n}(x)\right) .
$$


Definition 5 (Discrete Jacobian matrix) The discrete Jacobian matrix of $f$ evaluated at point $x \in \mathbb{B}^{n}$ is the following $n \times n$ Boolean matrix

$$
f^{\prime}(x)=\left(f_{i j}(x)\right), \quad f_{i j}(x)=f_{i}\left(x^{j 1}\right) \oplus f_{i}\left(x^{j 0}\right) \quad(i, j \in[n]) .
$$

In the next definition, we represent $f^{\prime}(x)$ under the form of a directed graph, in order to use graph theoretic notions instead of matrix theoretical notions. In fact, we mainly focus on elementary directed cycles, that we simply call cycles in the following.

Definition 6 (Local interaction graph) The local interaction graph of $f$ evaluated at point $x \in \mathbb{B}^{n}$ is the directed graph $G f(x)$ defined by: the vertex set is $[n]$, and for all $i, j \in[n]$, there exists an arc $j \rightarrow i$ if and only if $f_{i j}(x)=1$.

The discrete Jacobian matrix of $f$ was first defined by Robert (1983), who also introduced the notion of Boolean eigenvalue. This material allowed Shih and Ho (1999) to state a combinatorial analog of the Jacobian conjecture: if $f$ has the property that, for each $x \in \mathbb{B}^{n}$, all the boolean eigenvalues of $f^{\prime}(x)$ are zero, then $f$ has a unique fixed point. This conjecture was proved by Shih and Dong (2005). Since Robert proved that all the boolean eigenvalues of $f^{\prime}(x)$ are zero if and only if $G f(x)$ has no cycle, the theorem of Shih and Dong can be stated as follows.

Theorem 2 (Shih and Dong (2005)) If $G f(x)$ has no cycle $\forall x \in \mathbb{B}^{n}$, then $f$ has a unique fixed point.

A short prove of this theorem, independent of Theorem 1, is given in appendix. In the following of this section, we show, using Theorem 1, that the condition "if $G f(x)$ has no cycle for all $x$ " can be weakened into a condition of the form "if there exists "few" point $x$ such that $G f(x)$ has a "short" cycle". The exact statement is given after the following proposition.

Proposition 1 If $f$ is even or odd, then for every $x \in \mathbb{B}^{n}$ the out-degree of each vertex of $G f(x)$ is odd. In particular, $G f(x)$ has a cycle.

Proof: The out-degree $d_{j}^{+}$of any vertex $j$ of $G f(x)$, which equals the number of ones in the $j$ th column of $f^{\prime}(x)$, is $d_{j}^{+}=\left\|f\left(x^{j 1}\right) \oplus f\left(x^{j 0}\right)\right\|=\left\|f(x) \oplus f\left(x \oplus e_{j}\right)\right\|$. Since

$$
\left\|f(x) \oplus f\left(x \oplus e_{j}\right)\right\|=\left\|(x \oplus \tilde{f}(x)) \oplus\left(\left(x \oplus e_{j}\right) \oplus \tilde{f}\left(x \oplus e_{j}\right)\right)\right\|=\left\|\tilde{f}(x) \oplus \tilde{f}\left(x \oplus e_{j}\right) \oplus e_{j}\right\|,
$$

the parity of $d_{j}^{+}$is the parity of $\|\tilde{f}(x)\|+\left\|\tilde{f}\left(x \oplus e_{j}\right)\right\|+1$. Hence, if $f$ is even or odd, then $\|\tilde{f}(x)\|+$ $\left\|\tilde{f}\left(x \oplus e_{j}\right)\right\|$ is even, and $d_{j}^{+}$is odd.

Corollary 2 (Extension of Shih-Dong's fixed point theorem) If for $k=1, \ldots, n$, there exists at most $2^{k}-1$ points $x \in \mathbb{B}^{n}$ such that $G f(x)$ has a cycle of length at most $k$, then the conjugate of $f$ is a bijection. In particular, $f$ has a unique fixed point.

Proof: According to Theorem 1, it is sufficient to prove, by induction on $n$, that if $f$ satisfies the conditions of the statement, then $f$ has no even or odd self-dual subnetwork. The case $n=1$ is obvious. Suppose that $n>1$ and that $f$ satisfies the conditions of the statement. Let $i, j \in[n-1]$. For each $x \in \mathbb{B}^{n}$ such that $x_{n}=0$, we have

$$
f_{i j}^{n 0}\left(x_{-n}\right)=f_{i}^{n 0}\left(x_{-n}^{j 1}\right) \oplus f_{i}^{n 0}\left(x_{-n}^{j 0}\right)=f_{i}\left(x^{j 1}\right) \oplus f_{i}\left(x^{j 0}\right)=f_{i j}(x) .
$$


So $G f^{n 0}\left(x_{-n}\right)$ is the subgraph of $G f(x)$ induced by $[n-1]$, and we deduce that $f^{n \alpha}$ satisfies the condition of the theorem (for every $k \in[n-1]$, there exists at most $2^{k}-1$ points $x \in \mathbb{B}^{n-1}$ such that $G f^{n 0}(x)$ has a cycle of length at most $k$ ). Thus, by induction hypothesis, $f^{n 0}$ has no even or odd self-dual subnetwork. More generally, we prove with similar arguments, that for all $i \in[n], f^{i 0}$ and $f^{i 1}$ have no even or odd self-dual subnetwork. So $f$ has no odd or even self-dual strict subnetwork. If $f$ is itself even or odd self-dual, then by Proposition 1 . $G f(x)$ has a cycle for every $x \in \mathbb{B}^{n}$, so $f$ does not satisfy that conditions of the statement (for $k=n$ ). Therefore, $f$ has no even or odd self-dual subnetwork.

Example 2 (Continuation of Example 1) Take again

$$
f\left(x_{1}, x_{2}, x_{3}\right)=\left(\overline{x_{2}} \wedge x_{3}, \overline{x_{3}} \wedge x_{1}, \overline{x_{1}} \wedge x_{2}\right) .
$$

We have seen that $f$ has no self-dual subnetwork. So it satisfies the conditions of Theorem 1 but not the conditions of Shih-Dong's theorem, since $G f(000)$ and $G f(111)$ have a cycle:

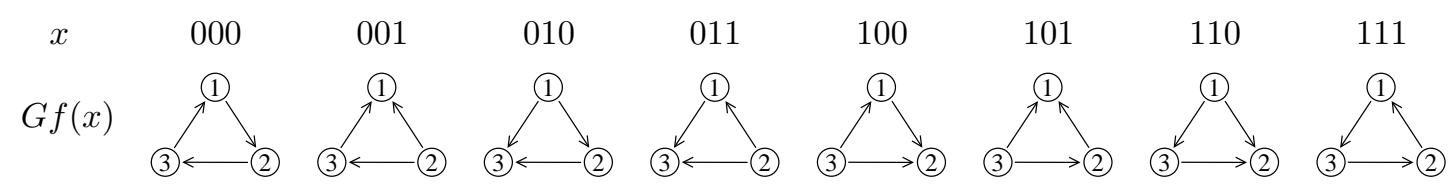

However, $f$ satisfies the condition of Corollary 2 (there is $0<2^{1}$ point $x$ with a cycle of length at most 1 ; $0<2^{2}$ point $x$ such that $G f(x)$ has a cycle of length at most 2 , and $2<2^{3}$ points $x$ such that $G f(x)$ has a cycle of length at most 3 ).

Now, consider the following "extension" $h: \mathbb{B}^{5} \rightarrow \mathbb{B}^{5}$ of $f$ :

$$
h\left(x_{1}, x_{2}, x_{3}, x_{4}, x_{5}\right)=\left(\overline{x_{2}} \wedge x_{3}, \overline{x_{3}} \wedge x_{1}, \overline{x_{1}} \wedge x_{2}, 0,0\right)=\left(f\left(x_{1}, x_{2}, x_{3}\right), 0,0\right)
$$

Using the fact that $f$ has no self-dual subnetwork, it's easy to see that $h$ has no self-dual subnetwork. So $h$ satisfies the conditions of Theorem 11 But it does not satisfy the conditions of Corollary 2 Indeed, there exists $2^{3}$ points $x$ such that $G h(x)$ has a cycle of length at most 3 :

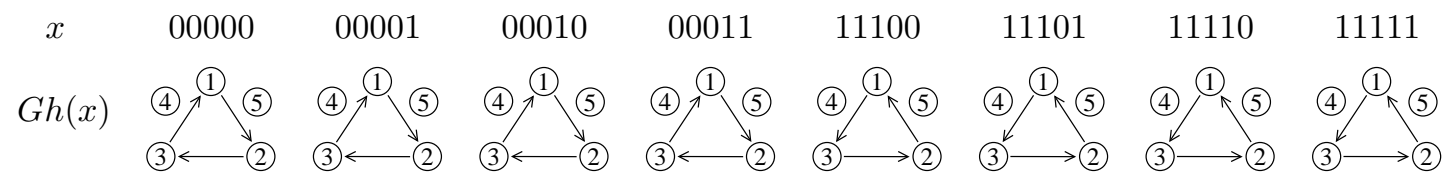

\section{Remarks on asynchronous state graphs}

In the following definition, we associate with $f: \mathbb{B}^{n} \rightarrow \mathbb{B}^{n}$ a directed graph on $\mathbb{B}^{n}$, called the asynchronous state graph of $f$, which has been proposed by Thomas (1973) as a model for the dynamics of gene networks; see also Thomas and d'Ari (1990).

Definition 7 (Asynchronous state graphs) The asynchronous state graph of $f$ is the directed graph $\Gamma(f)$ defined by: the vertex set is $\mathbb{B}^{n}$, and for every $x, y \in \mathbb{B}^{n}$, there exists an arc $x \rightarrow y$ if and only if there exists $i \in[n]$ such that $y=x \oplus e_{i}$ and $f_{i}(x) \neq x_{i}$. 
Remark that $\Gamma(f)$ and $f$ share the same information. Remark also that for every $i \in[n]$ and $\alpha \in \mathbb{B}$, $\Gamma\left(f^{i \alpha}\right)$ is isomorphic to the subgraph of $\Gamma(f)$ induced by the set of points $x \in \mathbb{B}^{n}$ such that $x_{i}=\alpha$. Indeed: for every $x, y \in \mathbb{B}^{n}$,

$$
\begin{aligned}
x_{-i} \rightarrow y_{-i} \text { is an } \operatorname{arc} \text { of } \Gamma\left(f^{i \alpha}\right) & \Longleftrightarrow \exists j \neq i \text { such that } y_{-i}=x_{-i} \oplus e_{j} \text { and } f_{j}^{i \alpha}\left(x_{-i}\right) \neq x_{j} \\
& \Longleftrightarrow \exists j \neq i \text { such that } y^{i \alpha}=x^{i \alpha} \oplus e_{j} \text { and } f_{j}\left(x^{i \alpha}\right) \neq x_{j} \\
& \Longleftrightarrow x^{i \alpha} \rightarrow y^{i \alpha} \text { is an arc of } \Gamma(f) .
\end{aligned}
$$

Corollary 3 If $f$ has no even or odd self-dual subnetwork, then $f$ has a unique fixed point $x$, and for all $y \in \mathbb{B}^{n}, \Gamma(f)$ contains a directed path from $y$ to $x$ of length $\|x \oplus y\|$.

By the definition of $\Gamma(f)$, a path from $x$ to $y$ cannot be of length strictly less than $\|x \oplus y\|$; a path from $x$ to $y$ of length $\|x \oplus y\|$ can thus be seen has a shortest path.

Proof of Corollary 3: by induction on $n$. The case $n=1$ is obvious, so suppose that $n>1$ and that the corollary holds for the dimensions less than $n$. Let $f: \mathbb{B}^{n} \rightarrow \mathbb{B}^{n}$, and suppose that $f$ has no even or odd self-dual subnetwork. By Theorem $1, f$ has a unique fixed point $x$. Let $y \in \mathbb{B}^{n}$. Suppose first that there exists $i \in[n]$ such that $x_{i}=y_{i}=0$. Then $x_{-i}$ is the unique fixed point of $f^{i 0}$. So, by induction hypothesis, $\Gamma\left(f^{i 0}\right)$ has a path from $y_{-i}$ to $x_{-i}$ of length $\left\|x_{-i} \oplus y_{-i}\right\|$. Since $x_{i}=y_{i}=0$, we deduce from $\star$ that $\Gamma(f)$ has a path from $y$ to $x$ of length $\left\|x_{-i} \oplus y_{-i}\right\|=\|x \oplus y\|$. The case $x_{i}=y_{i}=1$ is similar. So, finally, suppose that $y=x \oplus 1$. Since $y$ is not a fixed point, there exists $i \in[n]$ such that $f_{i}(y) \neq y_{i}$. Then, $\Gamma(f)$ has an arc from $y$ to $z=y \oplus e_{i}$. So $z_{i}=x_{i}$, and as previously, we deduce that $\Gamma(f)$ has a path from $z$ to $x$ of length $\|x \oplus z\|$. This path together with the arc $y \rightarrow z$ forms a path from $y$ to $x$ of length $\|x \oplus z\|+1=\|x \oplus y\|$.

According to (太), the asynchronous state graph of each subnetwork of $f$ is a subgraph of asynchronous state graph of $f$ induced by an hypercube contained in $\mathbb{B}^{n}$. Hence, one can see the asynchronous state graphs of the subnetworks of $f$ as "dynamical modules" of asynchronous state graph of $f$. The previous corollary shows that if $f$ has no even or odd self-dual subnetwork, then the asynchronous state graph of $f$ is "simple": it describes a "weak convergence" toward a unique fixed point. An interpretation is then that the asynchronous state graphs of even and odd self-dual networks are "dynamical modules" that are necessary for the "emergence" of "complex" asynchronous behaviors.

Example 3 (Continuation of Example 1) Take again the 3-dimensional network $f$ defined in Example 1 . which has no self-dual subnetwork. The asynchronous state graph $\Gamma(f)$ of $f$ is the following:

\begin{tabular}{ccc}
$x$ & $f(x)$ & $\tilde{f}(x)$ \\
\hline 000 & 000 & 000 \\
001 & 100 & 101 \\
010 & 001 & 011 \\
011 & 001 & 010 \\
100 & 010 & 110 \\
101 & 100 & 001 \\
110 & 010 & 100 \\
111 & 000 & 111
\end{tabular}

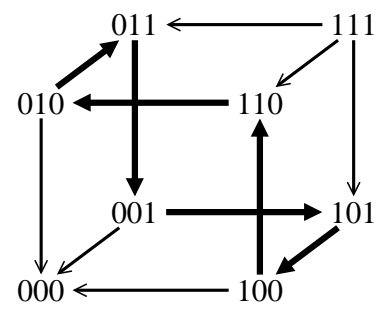


In agreement with Corollary 3 there exists, from any initial point, a shortest path leading to the unique fixed point of $f$ (the point 000): the asynchronous state graph describes a "weak asynchronous convergence" (by shortest paths) toward a unique fixed point. However, $\Gamma(f)$ has a cycle (of length 6$)$, so every path does not lead to the unique fixed point: the condition "has no even or odd self-dual subnetworks" does no ensure a "strong asynchronous convergence" toward a unique fixed point.

\section{Remarks on positive and negative cycles}

In this section, we show that positive (negative) circular networks, i.e. Boolean networks whose the global interaction graph reduces to a positive (negative) cycle, are simple instances of even (odd) circular networks. From this fact and existing results about positive and negative cycles, we will see that natural ideas of generalizations of Theorem 1 arise, but that none of these generalizations is true.

Let us begin with additional definitions. A signed directed graph is a directed graph in which each arc is either positive, negative or unsigned. In such a graph, a cycle is positive (negative) if it contains an unsigned arc or an even (odd) number of negative arcs (a directed cycle may be both positive and negative).

Definition 8 (Global interaction graph) The global interaction graph of $f: \mathbb{B}^{n} \rightarrow \mathbb{B}^{n}$ is the signed directed graph $G(f)$ defined by: the vertex set is $[n]$, and for all $i, j \in[n]$, there exists an arc $i \rightarrow j$ if and only if $f_{i}\left(x^{j 1}\right) \neq f_{i}\left(x^{j 0}\right)$ for at least one $x \in \mathbb{B}^{n}$; and an arc $j \rightarrow i$ of $G(f)$ is: positive if $f_{i}\left(x^{j 1}\right) \geq f_{i}\left(x^{j 0}\right)$ for all $x \in \mathbb{B}^{n}$; negative if $f_{i}\left(x^{j 1}\right) \leq f_{i}\left(x^{j 0}\right)$ for all $x \in \mathbb{B}^{n}$; and unsigned in the other cases.

Remark that $G(f)$ has an arc $j \rightarrow i$ if and only if $f_{i}$ depends on the $j$ th variable $x_{j}$ (and that $f_{i}\left(x^{j 1}\right) \neq$ $f_{i}\left(x^{j 0}\right)$ if and only if $\left.f_{i j}(x)=1\right)$.

Definition 9 (Positive and negative circular networks) $f$ is a positive (negative) circular network if $G(f)$ is a positive (negative) cycle.

The dynamics of positive and negative circular networks has been widely studied; see Remy et al. (2003) and Demongeot et al. (2010). Here, we prove that they are simple instances of even and odd self-dual networks.

Proposition 2 Every positive (negative) circular network is even (odd) and self-dual.

Proof: Let $f$ be a circular network. Without loss of generality, suppose that the $n$ arcs of $G(f)$ are $i+1 \rightarrow i$ for all $i \in[n] ; n+1$ being identified to 1 (here and in the rest of the proof). Then $f_{i}$ depends only on $x_{i+1}$, so either $f_{i}(x)=x_{i+1}$ (and $i+1 \rightarrow i$ is positive), or $f_{i}(x)=x_{i+1} \oplus 1$ (and $i+1 \rightarrow i$ is negative); in the first case, we set $s_{i}=0$, and in the second case, we set $s_{i}=1$ (so that $f_{i}(x)=x_{i+1} \oplus s_{i}$ in both cases). Let $s=\left(s_{1}, \ldots, s_{n}\right) \in \mathbb{B}^{n}$. By construction, $f$ is positive if $\|s\|$ is even, and negative if $\|s\|$ is odd. Furthermore,

$$
f(x)=\left(x_{2}, x_{3}, \ldots, x_{n}, x_{1}\right) \oplus s \quad \forall x \in \mathbb{B}^{n} .
$$

Hence

$$
f(x \oplus 1)=\left(x_{2} \oplus 1, \ldots, x_{n} \oplus 1, x_{1} \oplus 1\right) \oplus s=\left(x_{2}, \ldots, x_{n}, x_{1}\right) \oplus 1 \oplus s=f(x) \oplus 1
$$


So $f$ is self-dual. Also, we have $\tilde{f}(x)=x \oplus\left(x_{2}, \ldots, x_{n}, x_{1}\right) \oplus s$ so the parity of $\tilde{f}(x)$ is the parity of $\|x\|+\left\|\left(x_{2}, \ldots, x_{n}, x_{1}\right)\right\|+\|s\|$. Since $\|x\|=\left\|\left(x_{2}, \ldots, x_{n}, x_{1}\right)\right\|$, we deduce that the parity of $\tilde{f}(x)$ is the parity of $\|s\|$. So if $f$ is positive (negative) then the image of $\tilde{f}$ only contains even (odd) points.

It remains to prove that if $f$ is positive (negative) then each even (odd) point is in the image of $\tilde{f}$. Suppose that $f$ is positive (negative), and let $z$ be an even (odd) point of $\mathbb{B}^{n}$. Let $x \in \mathbb{B}^{n}$ be recursively defined by

$$
x_{1}=z_{n}, \quad x_{i+1}=z_{i} \oplus s_{i} \oplus x_{i} \quad \text { for all } i \in[n-1] .
$$

Then, for every $i \in[n-1]$, we have

$$
\tilde{f}_{i}(x)=x_{i} \oplus f_{i}(x)=x_{i} \oplus x_{i+1} \oplus s_{i}=x_{i} \oplus\left(z_{i} \oplus s_{i} \oplus x_{i}\right) \oplus s_{i}=z_{i} .
$$

If remains to prove that $\tilde{f}_{n}(x)=z_{n}$. By the definition of $x$, we have

$$
\begin{aligned}
x_{n} & =\left(z_{n-1} \oplus s_{n-1}\right) \oplus x_{n-1} \\
& =\left(z_{n-1} \oplus s_{n-1}\right) \oplus\left(z_{n-2} \oplus s_{n-2}\right) \oplus x_{n-2} \\
& \vdots \\
& =\left(z_{n-1} \oplus s_{n-1}\right) \oplus\left(z_{n-2} \oplus s_{n-2}\right) \oplus \cdots \oplus\left(z_{1} \oplus s_{1}\right) \oplus z_{n} \\
& =\left(z_{1} \oplus z_{2} \oplus \cdots \oplus z_{n}\right) \oplus\left(s_{1} \oplus s_{2} \oplus \cdots \oplus s_{n-1}\right) .
\end{aligned}
$$

So $z$ and $\left(s_{1}, s_{2}, \ldots, s_{n-1}, x_{n}\right)$ have the same parity, and since $z$ and $s$ have the same parity, we deduce that $x_{n}=s_{n}$. Thus $\tilde{f}_{n}(x)=x_{n} \oplus f_{n}(x)=s_{n} \oplus x_{1} \oplus s_{n}=x_{1}=z_{n}$, and we deduce that $\tilde{f}(x)=z$. So $f$ is even (odd) self-dual.

Remark 1 There are $2^{n-1}$ ! $n$-dimensional even (odd) self-dual networks, but "only" $(n-1) ! 2^{n-1} n$ dimensional positive (negative) circular networks. Since $2^{n-1} !=(n-1) ! 2^{n-1}$ for $n=1,2$, we deduce that every one or two-dimensional even (odd) self-dual network is a positive (negative) circular network.

Since the class of positive and negative circular networks is contained in the class of even and odd selfdual networks, it is natural to think about the following generalization of Theorem 1 . if $f$ has no positive or negative circular networks, then $f$ has a unique fixed point. However, this is false, as showed by the following example. Hence, Theorem 1 becomes false if "has no even or odd self-dual subnetwork" is replaced by "has no positive or negative circular subnetwork".

Example $4 f: \mathbb{B}^{4} \rightarrow \mathbb{B}^{4}$ is defined by

$$
\begin{aligned}
& f_{1}(x)=\left(\overline{x_{2}} \wedge x_{3} \wedge \overline{x_{4}}\right) \vee\left(\left(\overline{x_{2}} \vee x_{3}\right) \wedge x_{4}\right) \\
& f_{2}(x)=\left(\overline{x_{3}} \wedge x_{1} \wedge \overline{x_{4}}\right) \vee\left(\left(\overline{x_{3}} \vee x_{1}\right) \wedge x_{4}\right) \\
& f_{3}(x)=\left(\overline{x_{1}} \wedge x_{2} \wedge \overline{x_{4}}\right) \vee\left(\left(\overline{x_{1}} \vee x_{2}\right) \wedge x_{4}\right) \\
& f_{4}(x)=\left(x_{2} \wedge x_{3} \wedge \overline{x_{1}}\right) \vee\left(\left(x_{2} \vee x_{3}\right) \wedge x_{1}\right)
\end{aligned}
$$


The table of $f$ and $\tilde{f}$, and the asynchronous state graph of $f$ are as follow:

\begin{tabular}{ccc}
$x$ & $f(x)$ & $\tilde{f}(x)$ \\
\hline 0000 & 0000 & 0000 \\
0010 & 1000 & 1010 \\
0100 & 0010 & 0110 \\
0110 & 0011 & 0101 \\
1000 & 0100 & 1100 \\
1010 & 1001 & 0011 \\
1100 & 0101 & 1001 \\
1110 & 0001 & 1111 \\
0001 & 1110 & 1111 \\
0011 & 1010 & 1001 \\
0101 & 0110 & 0011 \\
0111 & 1011 & 1100 \\
1001 & 1100 & 0101 \\
1011 & 1101 & 0110 \\
1101 & 0111 & 1010 \\
1111 & 1111 & 0000
\end{tabular}

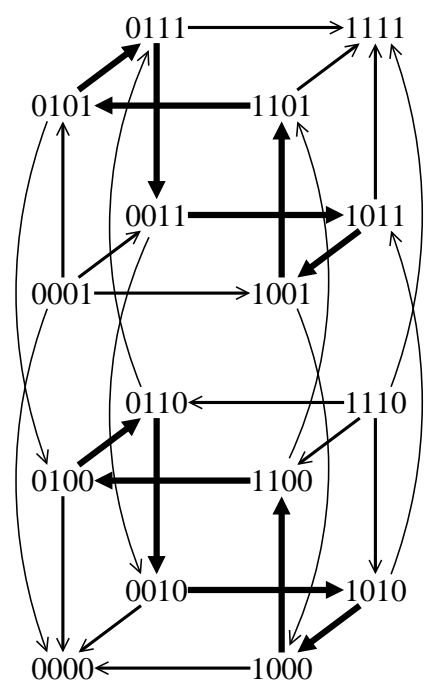

One can see that $f$ is even self-dual. The immediate subnetworks of $f$ are the following:

$$
\begin{aligned}
& f^{10}\left(x_{2}, x_{3}, x_{4}\right)=\left(\overline{x_{3}} \wedge x_{4}, x_{2} \vee x_{4}, x_{2} \wedge x_{3}\right) \\
& f^{11}\left(x_{2}, x_{3}, x_{4}\right)=\left(\overline{x_{3}} \vee x_{4}, x_{2} \wedge x_{4}, x_{2} \vee x_{3}\right) \\
& f^{20}\left(x_{1}, x_{3}, x_{4}\right)=\left(x_{3} \vee x_{4}, \overline{x_{1}} \wedge x_{4}, x_{3} \wedge x_{1}\right) \\
& f^{21}\left(x_{1}, x_{3}, x_{4}\right)=\left(x_{3} \wedge x_{4}, \overline{x_{1}} \vee x_{4}, x_{3} \vee x_{1}\right) \\
& f^{30}\left(x_{1}, x_{2}, x_{4}\right)=\left(\overline{x_{2}} \wedge x_{4}, x_{1} \vee x_{4}, x_{2} \wedge x_{1}\right) \\
& f^{31}\left(x_{1}, x_{2}, x_{4}\right)=\left(\overline{x_{2}} \vee x_{4}, x_{1} \wedge x_{4}, x_{2} \vee x_{1}\right) \\
& f^{40}\left(x_{1}, x_{2}, x_{3}\right)=\left(\overline{x_{2}} \wedge x_{3}, \overline{x_{3}} \wedge x_{1}, \overline{x_{1}} \wedge x_{2}\right) \quad \text { (as in Examples 1-3) } \\
& f^{41}\left(x_{1}, x_{2}, x_{3}\right)=\left(\overline{x_{2}} \vee x_{3}, \overline{x_{3}} \vee x_{1}, \overline{x_{1}} \vee x_{2}\right)
\end{aligned}
$$

Proceeding as in Example 1, one can check that none immediate subnetwork of $f$ has a self-dual subnetwork (actually, it is sufficient to check this for each $f^{i 0}$ since $\left.f^{i 1}(x)=f^{i 0}(x \oplus 1) \oplus 1,1 \leq i \leq 4\right)$. So $f$ has no circular strict subnetwork, and since $f$ is not circular, $f$ has no circular subnetwork, but it has not a unique fixed points. Note that for $1 \leq i \leq 4$, the 4-dimensional network $h$ defined by $h(x)=f(x) \oplus e_{i}$ is odd self-dual, has no circular subnetwork, and no fixed point.

Now, consider the following three fundamental theorems about cycles and fixed points (the last two theorems result from two conjectures of Thomas; see Remy et al. (2008); Richard (2010) and the references therein).

Theorem 3 (Robert(1980)) If $G(f)$ has no cycle, then $f$ has a unique fixed point.

Remark 2 Clearly, each local interaction graph $G f(x)$ is a subgraph of the (unsigned version of the) global interaction graph $G(f)$. Hence, the condition " $G(f)$ has no cycle" of Robert's theorem is (much 
more) stronger than the condition " $G f(x)$ has no cycle for every $x$ " of Shih-Dong's Theorem. Consequently, Shih-Dong's theorem is a generalization of Robert's theorem. Thus, Theorem 1 is also a generalization of Robert's theorem.

Remark 3 Actually, Robert proved, in Robert (1980) and Robert (1995), that if $G(f)$ has no cycle, then $f$ has a unique fixed point $x$ and: (1) the synchronous iteration $x^{t+1}=f\left(x^{t}\right)$ converges toward $x$ in at most $n$ steps for every initial point $x^{0} \in \mathbb{B}^{n} ;(2)$ every path of $\Gamma(f)$ leads to $x$ in at most $n$ steps ("strong asynchronous convergence by shortest paths toward a unique fixed points"). These results shows the necessity of cycles for obtaining "complex" synchronous or asynchronous behaviors (e.g. multiple fixed points, cyclic attractors, long transient phases...).

Theorem 4 (Remy et al.(2008)) If $G(f)$ has no positive cycle, then $f$ has at most one fixed point.

Remark 4 Actually, by saying that an arc $j \rightarrow i$ of $G f(x)$ is positive if $f_{i}\left(x^{j 1}\right)>f_{i}\left(x^{j 0}\right)$ and negative if $f_{i}\left(x^{j 1}\right)<f_{i}\left(x^{j 0}\right)$, Remy et al. (2008) proved the following more general statement: if $G f(x)$ has no positive cycle for all $x \in \mathbb{B}^{n}$, then $f$ has at most one fixed point.

Theorem 5 (Richard (2010)) If $G(f)$ has no negative cycle, then $f$ has at least one fixed point.

Hence, Theorems 4 and 5 give a nice proof "by dichotomy" of Robert's theorem: the absence of positive cycle gives the uniqueness, and absence of negative cycle gives the existence. Seeing the relationship between positive (negative) circular networks and even (odd) self-dual networks, one may ask if a "proof by dichotomy" occurs for Theorem 11, i.e., if the absence of even self-dual subnetwork gives the uniqueness, and if the absence of odd self-dual network gives the existence. The following example shows that both cases are false. Hence: if $f$ has no even (odd) self-dual subnetworks, than it has not necessarily at most (at least) one fixed point.

Example $5 f: \mathbb{B}^{3} \rightarrow \mathbb{B}^{3}$ is defined by

$$
\begin{aligned}
& f_{1}(x)=\left(\overline{x_{1}} \wedge\left(x_{2} \vee x_{3}\right)\right) \vee\left(x_{2} \wedge x_{3}\right) \\
& f_{2}(x)=\left(\overline{x_{2}} \wedge\left(x_{3} \vee x_{1}\right)\right) \vee\left(x_{3} \wedge x_{1}\right) \\
& f_{3}(x)=\left(\overline{x_{3}} \wedge\left(x_{1} \vee x_{2}\right)\right) \vee\left(x_{1} \wedge x_{2}\right)
\end{aligned}
$$

The table of $f$ and $\tilde{f}$, and the asynchronous state graph of $f$ are as follow:

\begin{tabular}{ccc}
$x$ & $f(x)$ & $\tilde{f}(x)$ \\
\hline 000 & 000 & 000 \\
001 & 110 & 111 \\
010 & 101 & 111 \\
011 & 100 & 111 \\
100 & 011 & 111 \\
101 & 010 & 111 \\
110 & 001 & 111 \\
111 & 111 & 000
\end{tabular}

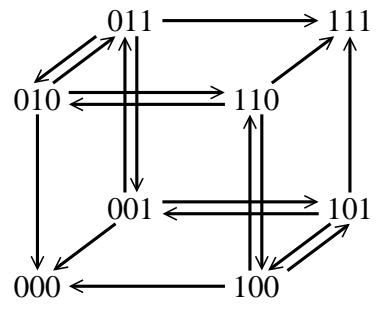


$f$ is self-dual, but not even since $\|f(001)\|$ is odd. The immediate subnetworks of $f$ are:

$$
\begin{aligned}
& f^{10}\left(x_{2}, x_{3}\right)=\left(\overline{x_{2}} \wedge x_{3}, \overline{x_{3}} \wedge x_{2}\right) \\
& f^{11}\left(x_{2}, x_{3}\right)=\left(\overline{x_{2}} \vee x_{3}, \overline{x_{3}} \vee x_{2}\right) \\
& f^{20}\left(x_{1}, x_{3}\right)=\left(\overline{x_{1}} \wedge x_{3}, \overline{x_{3}} \wedge x_{1}\right) \\
& f^{21}\left(x_{1}, x_{3}\right)=\left(\overline{x_{1}} \vee x_{3}, \overline{x_{3}} \vee x_{1}\right) \\
& f^{30}\left(x_{1}, x_{2}\right)=\left(\overline{x_{1}} \wedge x_{2}, \overline{x_{2}} \wedge x_{1}\right) \\
& f^{31}\left(x_{1}, x_{2}\right)=\left(\overline{x_{1}} \vee x_{2}, \overline{x_{2}} \vee x_{1}\right)
\end{aligned}
$$

So each $f^{i \alpha}$ is not circular, and according to Remark 1 it is not even and self-dual. Furthermore, each strict subnetwork $h$ of $f^{i \alpha}$ is either constant or defined by $h(0)=1$ and $h(1)=0$ (in the second case, $h$ is odd and self-dual). So $f^{i \alpha}$ has no strict even self-dual subnetwork. We deduce that $f$ has no even self-dual subnetwork. But it has two fixed points.

Now consider the network $f: \mathbb{B}^{3} \rightarrow \mathbb{B}^{3}$ is defined by

$$
\begin{aligned}
& f_{1}(x)=\overline{x_{2}} \\
& f_{2}(x)=\overline{x_{3}} \\
& f_{3}(x)=\left(x_{3} \wedge\left(\overline{x_{1}} \vee x_{2}\right)\right) \vee\left(\overline{x_{1}} \wedge x_{2}\right)
\end{aligned}
$$

The table of $f$ and $\tilde{f}$, and the asynchronous state graph of $f$ are as follow:

\begin{tabular}{ccc}
$x$ & $f(x)$ & $\tilde{f}(x)$ \\
\hline 000 & 110 & 110 \\
001 & 101 & 100 \\
010 & 011 & 001 \\
011 & 001 & 010 \\
100 & 110 & 010 \\
101 & 100 & 001 \\
110 & 010 & 100 \\
111 & 001 & 110
\end{tabular}

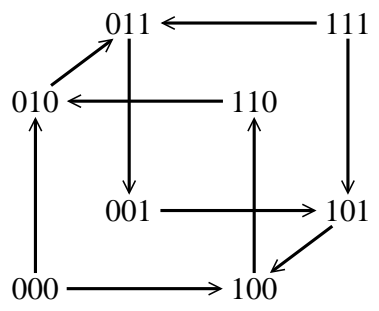

$f$ is self-dual, but not odd since $\|f(000)\|$ is even. The immediate subnetworks of $f$ are:

$$
\begin{aligned}
& f^{10}\left(x_{2}, x_{3}\right)=\left(\overline{x_{3}}, x_{3} \vee x_{2}\right) \\
& f^{11}\left(x_{2}, x_{3}\right)=\left(\overline{x_{3}}, x_{3} \wedge x_{2}\right) \\
& f^{20}\left(x_{1}, x_{3}\right)=\left(1, x_{3} \wedge \overline{x_{1}}\right) \\
& f^{21}\left(x_{1}, x_{3}\right)=\left(0, x_{3} \vee \overline{x_{1}}\right) \\
& f^{30}\left(x_{1}, x_{2}\right)=\left(\overline{x_{2}}, 1\right) \\
& f^{31}\left(x_{1}, x_{2}\right)=\left(\overline{x_{2}}, 0\right)
\end{aligned}
$$

So each $f^{i \alpha}$ is not circular, and according to Remark 1 it is not odd and self-dual. Furthermore, each strict subnetwork $h$ of $f^{i \alpha}$ is either constant or defined by $h(0)=0$ and $h(1)=1$ (in the second case, $h$ is even and self-dual). So $f^{i \alpha}$ has no strict odd self-dual subnetwork. We deduce that $f$ has no odd self-dual subnetwork. But it has no fixed point. 


\section{Acknowledgements}

I wish to thank Julie Boyon and Sebastien Brun for interesting discussions. This work has been partially supported by the French National Agency for Reasearch (ANR-10-BLANC-0218 BioTempo project).

\section{A A short proof of the theorem of Shih and Dong}

The "trick" consists in proving, by induction on $n$, the following more general statement:

(*) If $G f(x)$ has no cycle for all $x \in \mathbb{B}^{n}$, then the conjugate of $f$ is a bijection (so that $f$ has a unique fixed point).

The case $n=1$ is obvious, so suppose that $n>1$ and that $(*)$ holds for the dimensions less than $n$. Suppose that $G f(x)$ has no cycle for all $x \in \mathbb{B}^{n}$. Let $i, j \in[n-1]$, and $x \in \mathbb{B}^{n}$ such that $x_{n}=0$. We have

$$
f_{i j}^{n 0}\left(x_{-n}\right)=f_{i}^{n 0}\left(x_{-n}^{j 1}\right) \oplus f_{i}^{n 0}\left(x_{-n}^{j 0}\right)=f_{i}\left(x^{j 1}\right) \oplus f_{i}\left(x^{j 0}\right)=f_{i j}(x) .
$$

So $G f^{n 0}\left(x_{-n}\right)$ is the subgraph of $G f(x)$ induced by $[n-1]$, and thus, it has no cycle. We deduce that $f^{n 0}$ satisfies the conditions of $(*)$. Thus, by induction hypothesis, the conjugate of $f^{n 0}$ is a bijection. We prove with similar arguments that $\tilde{f}^{i 0}$ and $\tilde{f}^{i 1}$ are bijections for all $i \in[n]$.

Now, suppose, by contradiction, that $\tilde{f}$ is not a bijection. Then, there exists two distinct points $x, y \in \mathbb{B}^{n}$ such that $\tilde{f}(x)=\tilde{f}(y)$. Let us proved that $x=y \oplus 1$. Indeed, if $x_{i}=y_{i}=\alpha$ for some $i \in[n]$, then $\tilde{f}^{i \alpha}\left(x_{-i}\right)=\tilde{f}(x)_{-i}=\tilde{f}(y)_{-i}=\tilde{f}^{i \alpha}\left(y_{-i}\right)$. Thus $\tilde{f}^{i \alpha}$ is not a bijection, a contradiction. So $x=y \oplus 1$. Since $G f(x)$ has no cycle, it contains at least one vertex of out-degree 0 . In other words, there exists $i \in[n]$ such that $f\left(x^{i 1}\right)=f\left(x^{i 0}\right)$. Thus $\tilde{f}\left(x^{i 1}\right)_{-i}=\tilde{f}\left(x^{i 0}\right)_{-i}=\tilde{f}(x)_{-i}$. Hence, setting $\alpha=y_{i}$, we obtain

$$
\tilde{f}^{i \alpha}\left(x_{-i}\right)=\tilde{f}\left(x^{i \alpha}\right)_{-i}=\tilde{f}(x)_{-i}=\tilde{f}(y)_{-i}=\tilde{f}\left(y^{i 1}\right)_{-i}=\tilde{f}^{i 1}\left(y_{-i}\right) .
$$

So $\tilde{f}^{i \alpha}$ is not a bijection, a contradiction. Thus $\tilde{f}$ is a bijection and $(*)$ is proved.

\section{References}

J. Demongeot, M. Noual, and S. Sené. On the number of attractors of positive and negative Boolean automata circuits. In Proceedings of WAINA'10, pages 782-789. IEEE press, 2010.

S. A. Kauffman. Metabolic stability and epigenesis in randomly connected nets. Journal of Theoretical Biology, 22:437-467, 1969.

E. Remy, B. Mossé, C. Chaouiya, and D. Thieffry. A description of dynamical graphs associated to elementary regulatory circuits. Bioinformatics, 19:172-178, 2003.

E. Remy, P. Ruet, and D. Thieffry. Graphic requirements for multistability and attractive cycles in a boolean dynamical framework. Advances in Applied Mathematics, 41(3):335 - 350, 2008. ISSN 01968858

A. Richard. Negative circuits and sustained oscillations in asynchronous automata networks. Advances in Applied Mathematics, 44(4):378 - 392, 2010. ISSN 0196-8858. 
F. Robert. Iterations sur des ensembles finis et automates cellulaires contractants. Linear Algebra and its Applications, 29:393-412, 1980.

F. Robert. Dérivée discrete et convergence locale d'une itération Booléenne. Linear Algebra Appl., 52: $547-589,1983$.

F. Robert. Les systèmes dynamiques discrets, volume 19 of Mathématiques et Applications. Springer, 1995.

M.-H. Shih and J.-L. Dong. A combinatorial analogue of the Jacobian problem in automata networks. Advances in Applied Mathematics, 34:30-46, 2005.

M.-H. Shih and J.-L. Ho. Solution of the Boolean Markus-Yamabe problem. Advances in Applied Mathematics, 22:60-102, 1999.

R. Thomas. Boolean formalization of genetic control circuits. Journal of Theoretical Biology, 42(3):563 - 585, 1973. ISSN 0022-5193.

R. Thomas and R. d'Ari. Biological Feedback. CRC Press, 1990. 
